\title{
Tratamento endovascular da oclusão de ramo ilíaco de endoprótese bifurcada de aorta abdominal: trombectomia rotativa e aspirativa seguida de angioplastia com stent primário
}

\author{
Endovascular treatment of iliac limb occlusion of a bifurcated abdominal aortic stent \\ graft - rotational and aspiration thrombectomy followed by primary angioplasty and stenting
}

Fabio Augusto Cypreste Oliveira', Fabio Lemos Campedelli', Carlos Eduardo de Sousa Amorelli', José Eduardo da Costa Filho², Daniel Resende Gibbon², Juliana Caetano Barreto ${ }^{3}$, Philippe Moreira da Silva ${ }^{4}$

\begin{abstract}
Resumo
A oclusão de ramo ilíaco de endoprótese bifurcada de aorta surge como complicação decorrente da correção endovascular do aneurisma de aorta abdominal e várias abordagens terapêuticas têm sido empregadas para o tratamento dessa complicação em casos de isquemia de membro inferior. Apresentamos dois casos de tratamento totalmente percutâneo da oclusão de ramo ilíaco de endoprótese de aorta abdominal com dispositivo de trombectomia rotativa e aspirativa seguida de angioplastia com stent primário, sem complicações operatórias.
\end{abstract}

Palavras-chave: oclusão de enxerto vascular; trombectomia; aneurisma.

\begin{abstract}
Iliac limb occlusion of bifurcated aortic stent graft appears as complication of endovascular repair of abdominal aortic aneurysm and several therapeutic approaches have been used to treat this problem in cases of lower limb ischemia. In two cases a totally endovascular approach was used to treat the limb occlusion by means of rotational and aspiration thrombectomy, followed by stenting. In both cases there were no postoperative complications.
\end{abstract}

Keywords: graft occlusion, vascular; thrombectomy; aneurysm.

\section{Introdução}

A correção endovascular do aneurisma de aorta abdominal (CEAAA) foi desenvolvida para um tratamento menos invasivo e com o objetivo de diminuir a morbi/mortalidade do tratamento cirúrgico em pacientes portadores de aneurisma de aorta. Ela tem se mostrado responsável pela redução significativa da taxa de mortalidade operatória, do volume de transfusão sanguínea e do tempo cirúrgico e de internação em unidade de terapia intensiva. Entretanto, requer maior número de intervenções secundárias para manutenção da exclusão aneurismática e/ou tratamento das complicações ${ }^{1-6}$.
A intervenção secundária é definida pela Society for Vascular Surgery (SVS) e pela American Association for Vascular Surgery (AAVS) como qualquer procedimento cirúrgico ou endovascular realizado após a correção endovascular inicial ${ }^{7}$.

O registro Eurostar ${ }^{8}$ classifica as intervenções secundárias de acordo com a sua magnitude: 1) necessidade de laparotomia; 2) procedimentos extra-anatômicos; 3 ) intervenções transfemorais percutâneas ou abertas; todos em busca da padronização para melhor conhecimento e terapia das complicações da CEAAA.

Trabalho realizado no Serviço de Cirurgia Vascular e Endovascular do Hospital Santa Teresa - Petrópolis (RJ), Brasil.

Trabalho apresentado no XI Panamerican Congress on Vascular and Endovascular Surgery, em novembro de 2010, no Rio de Janeiro (RI).

'Especialista em Cirurgia Vascular com área de atuação em Cirurgia Endovascular e Angiorradiologia pela Sociedade Brasileira de Angiologia e Cirurgia Vascular (SBACV) - Rio de Janeiro (RJ), Brasil.

Especialista em Cirurgia Vascular pela Sociedade Brasileira de Angiologia e Cirurgia Vascular (SBACV) - Rio de Janeiro (RJ), Brasil.

${ }^{3}$ Graduanda em Medicina pela Faculdade de Medicina de Petrópolis. Acadêmica concursada do Serviço de Terapia Intensiva do Hospital Santa Teresa - Petrópolis (RJ), Brasil.

${ }^{4}$ Técnico de Enfermagem e Instrumentador do Serviço de Cirurgia Vascular e Endovascular do Hospital Santa Teresa - Petrópolis (RJ), Brasil.

Fonte de financiamento: não há

Conflito de interesse: nada a declarar

Artigo submetido em: 24.08.11. Aceito em: 03.05 .12

J Vasc Bras. 2012;11(3):212-218. 
Atualmente as taxas de intervenção secundária após a correção estão diretamente relacionadas à experiência profissional, seleção morfológica do caso para CEAAA, remodelamento arterial, material utilizado, seguimento do paciente e diagnóstico das complicações (vazamentos, endotensão, migrações/desconexões, oclusões e roturas).

A oclusão de ramo ilíaco de endoprótese bifurcada de aorta pode ocorrer em todos os modelos e acontece em menos de $10 \%$ dos pacientes submetidos à correção $0^{9,10}$. Apresenta-se de forma mais precoce que os vazamentos (3,5 meses versus 14,3 meses) e, na maioria dos casos, requer intervenção por isquemia do membro afetado ${ }^{11}$. O número reduzido de casos, a ausência de estudos com resultados a longo prazo e a variedade do arsenal terapêutico com diferentes riscos e resultados (trombólise, trombectomia cirúrgica, angioplastia, procedimentos extra-anatômicos, entre outros) para o tratamento da oclusão do ramo ilíaco dificultam a definição do padrão ouro de abordagem terapêutica. Inicialmente as derivações extra-anatômicas eram definidas como tratamento de escolha, porém atualmente há tendência mundial na abordagem totalmente percutânea dessa complicação, mostrando resultados iniciais satisfatórios ${ }^{12-15}$.

\section{Descrição dos casos}

\section{Caso I}

Paciente masculino de 69 anos, apresentando como comorbidades hipertensão arterial sistêmica, diabetes mellitus e coronariopatia, foi submetido à CEAAA por aneurisma bissacular infrarrenal sintomático, com implante de endoprótese bifurcada de aorta Talent ${ }^{\circledast}$ (Medtronic Inc./USA) $26 \times 16 \times 105$ com fixação distal em ilíaca comum bilateralmente. Evoluiu no pós-operatório sem intercorrências e recebeu alta no $3^{\circ}$ dia de internação hospitalar (DIH), assintomático e com índice tornozelo braquial (ITB) 1,0 em ambos os membros inferiores.

Após 30 dias do procedimento inicial, apresentou quadro de dor súbita em membro inferior direito, evoluindo para claudicação incapacitante (Rutherford I-3). Com o exame físico identificou-se ausência de pulsos palpáveis nesse membro, ITB 0,6 e eco Doppler colorido com fluxo monofásico desde artéria femoral comum até artérias distais. Foi realizada angiotomografia, que confirmou a oclusão de segmento ilíaco direito livre de stent da endoprótese com reabitação ao nível da bifurcação ilíaca. Evidenciou-se compressão do segmento direito pelo ramo esquerdo pérvio, ausência de desconexões e manutenção da exclusão aneurismática (Figuras 1a e 1b).

Foi, então, realizado sob anestesia local e sedação, acesso percutâneo femoral bilateral com introdução de bainha 7F à direita e $6 \mathrm{~F}$ à esquerda, ultrapassada a lesão com fio guia hidrofílico $0,035 \times 260,000$ montado em microcateter hidrofílico $4 \mathrm{~F}$ seguido de aortografia. Realizou-se também trombectomia rotativa e aspirativa com dispositivo Rotarex ${ }^{\circledR}$ (Straubmedical Inc./CHE) 6F, seguida de angioplastia com stent autoexpansível $12 \mathrm{~mm}$ de diâmetro e pós-dilatação com cateter balão 10 $\mathrm{mm}$ de diâmetro à direita e $8 \mathrm{~mm}$ à esquerda (kissing baloon), de modo a evitar a compressão contralateral. A aortografia de controle demonstrou recanalização completa sem sinais de recuo elástico e/ou embolização distal (Figura 2).

$\mathrm{O}$ paciente apresentou evolução pós-operatória sem intercorrências e recebeu alta hospitalar no $3^{\circ} \mathrm{DIH}$ assintomático com todos os pulsos palpáveis e ITB 1,0 bilateral. Ao escrevermos este artigo, encontrava-se em acompanhamento ambulatorial com nove meses de evolução após a intervenção secundária para a recanalização do ramo ilíaco da endoprótese. Apresentava-se assintomático, com pulsos universalmente palpáveis e ITB 1,0 bilateral.

As angiotomografias de controle realizadas no primeiro, terceiro e sexto mês não demonstraram qualquer sinal de reestenose (Figura 3).

\section{Caso II}

Paciente masculino de 74 anos, apresentando como comorbidades hipertensão arterial sistêmica, diabetes mellitus, doença pulmonar obstrutiva crônica e coronariopatia, foi submetido à CEAAA por aneurisma fusiforme infrarrenal (maior diâmetro: 6,7 cm) com implante de endoprótese bifurcada de aorta Talent ${ }^{\oplus}$ (Medtronic Inc./USA) 28x16x105 com fixação distal em artéria ilíaca comum bilateralmente. Evoluiu no pós-operatório sem intercorrências e recebeu alta hospitalar no $3^{\circ} \mathrm{DIH}$ assintomático com ITB 1,0 bilateral.

$\mathrm{O}$ paciente permaneceu assintomático, sem particularidades no exame físico. As angiotomografias realizadas no primeiro, terceiro e sexto mês demonstravam exclusão do aneurisma, sem sinais de vazamento, desconexão ou compressão (Figura 4).

No nono mês de pós-operatório apresentou quadro de dor súbita em membro inferior direito seguido de claudicação incapacitante do mesmo (Rutherford I-3). No exame físico havia ausência de pulsos palpáveis nesse membro, ITB 0,68 e eco Doppler colorido com fluxo monofásico desde artéria femoral comum até artérias distais. Foi realizada, então, angiotomografia que identificou oclusão de ramo ilíaco direito da endoprótese com reabitação ao nível da bifurcação ilíaca. Observou-se mudança de angulação entre o segmento ilíaco da endoprótese e a artéria ilíaca externa reabitada, ausência de desconexões ou migrações e ausência de vazamentos (Figura 5). 

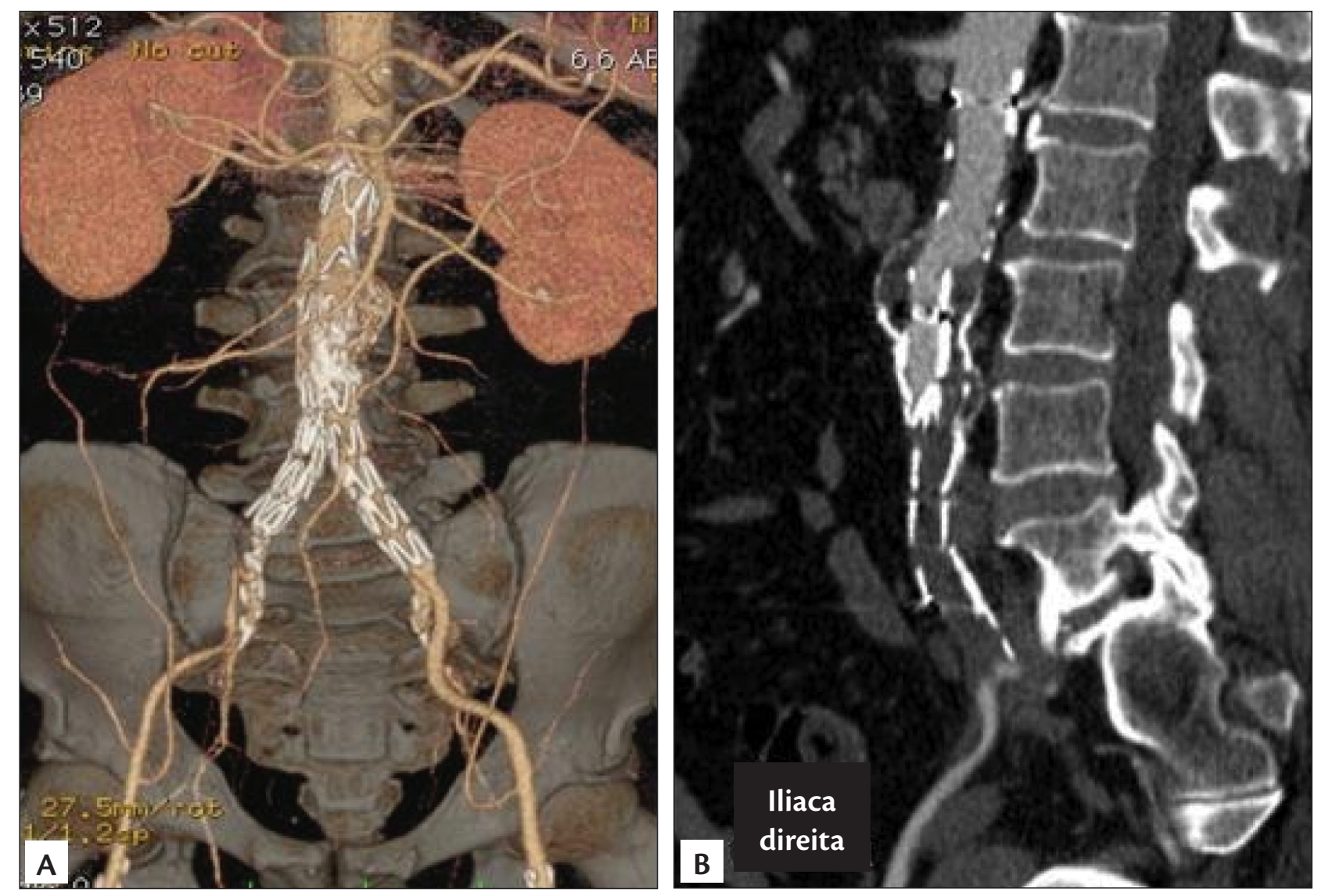

Figura 1. a) Angiotomografia pós-operatória mostrando oclusão do segmento ilíaco direito; b) Identificada compressão do segmento ilíaco livre de stent no corte sagital.

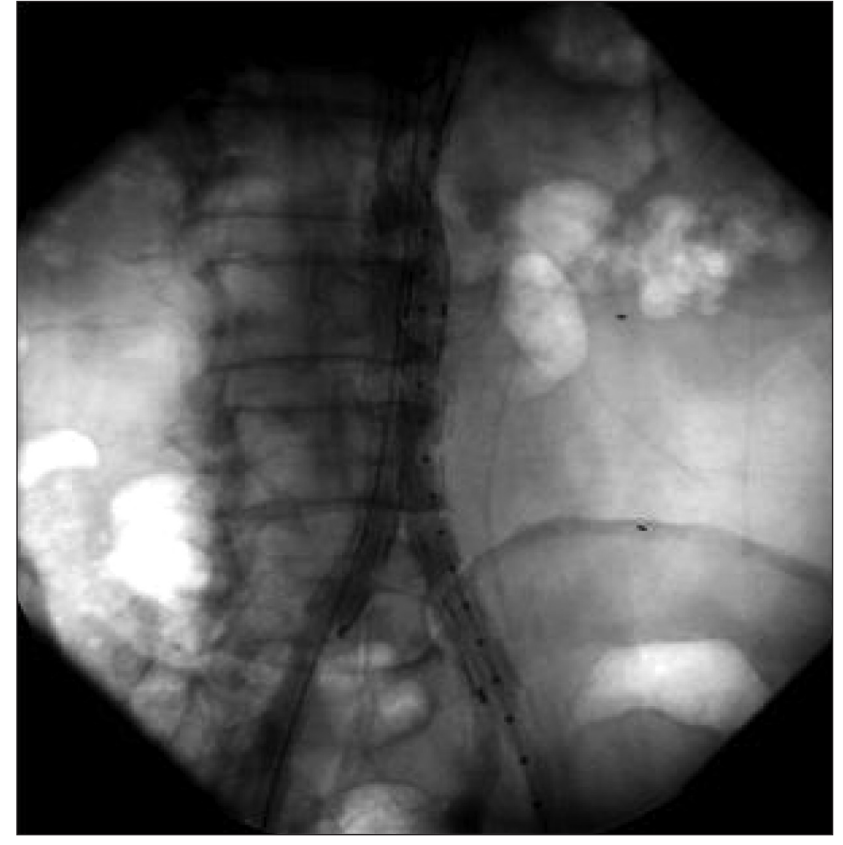

Figura 2. Aortografia de controle peroperatória com recanalização completa da oclusão.

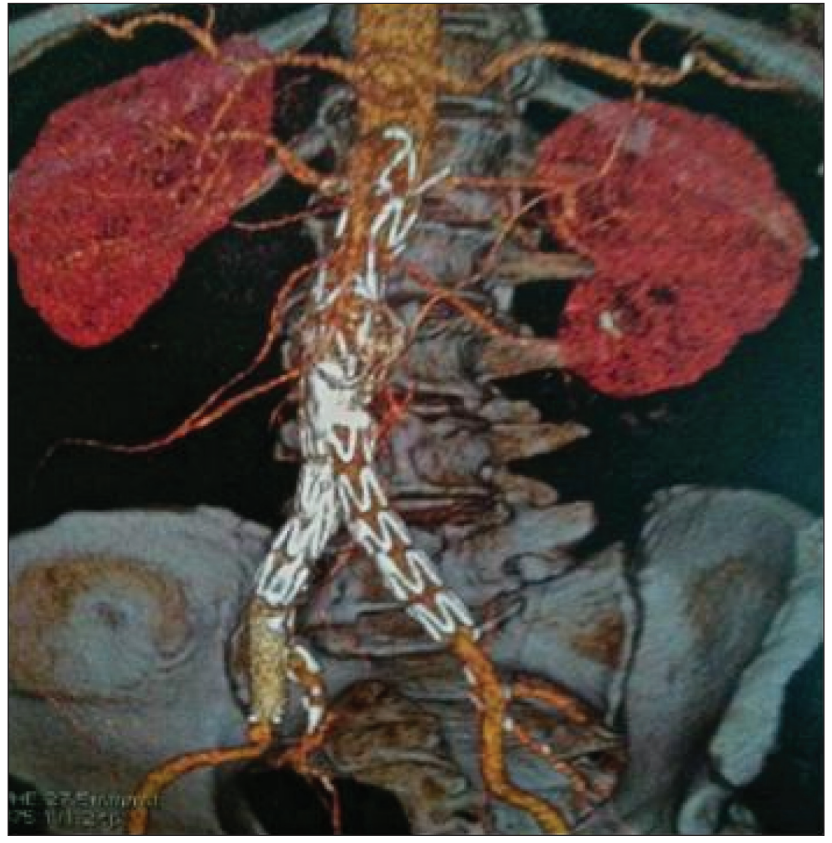

Figura 3. Angiotomografia de controle com perviedade do segmento ilíaco direito após implante do stent. 


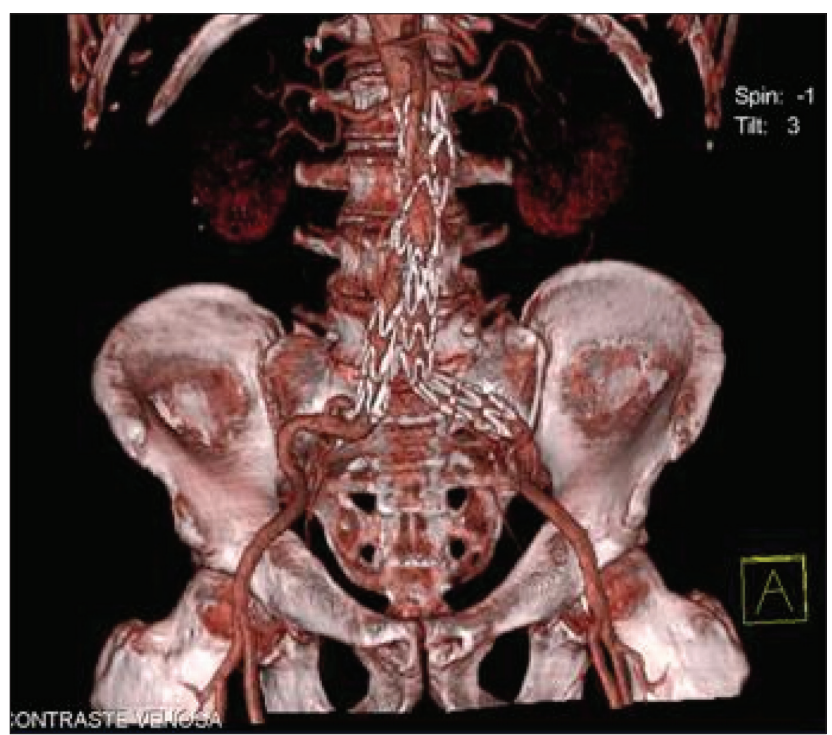

Figura 4. Angiotomografia de controle seis meses após procedimento inicial de correção endovascular do aneurisma de aorta abdominal.

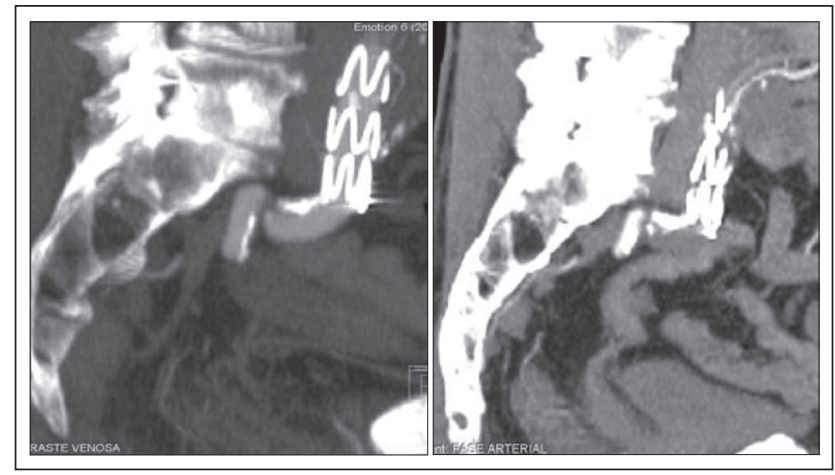

Figura 5. Angiotomografia em corte sagital mostrando mudança na angulação de fixação distal e trombose da endoprótese.

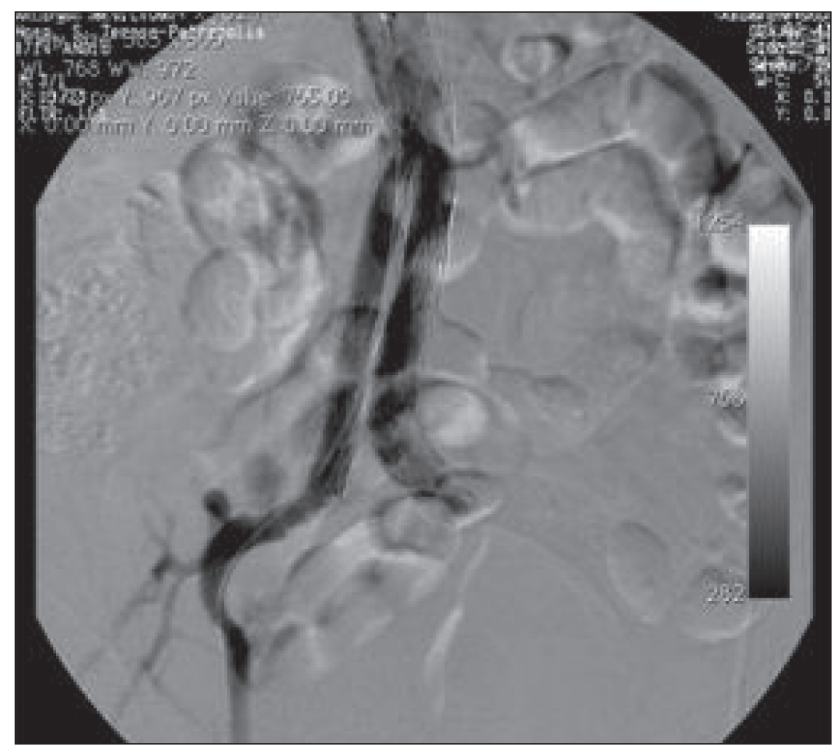

Figura 6. Resultado inicial pós-trombectomia e angioplastia após implante de stent Ddentro da endoprótese.
Foi, então, realizado sob anestesia local e sedação, acesso percutâneo braquial esquerdo com introdução de bainha 7F longa, ultrapassada a lesão com guia hidrofílico $0,035 \times 260,000$ montado em microcateter hidrofílico $4 \mathrm{~F}$ seguido de aortografia, evidenciando a oclusão. Realizou-se também trombectomia rotativa e aspirativa com dispositivo Rotarex $6 \mathrm{~F}^{\circ}$ (Straubmedical Inc./CHE) seguida de angioplastia com stent autoexpansível $12 \mathrm{~mm}$ de diâmetro cobrindo todo o segmento ocluído da endoprótese até artéria ilíaca externa direita e pós-dilatação com cateter balão $10 \mathrm{~mm}$ de diâmetro. A arteriografia de controle mostra recanalização completa da lesão e ausência de sinais de recuo elástico e/ou embolização distal (Figura 6).

O paciente apresentou evolução pós-operatória sem intercorrências e recebeu alta hospitalar no $3^{\circ} \mathrm{DIH}$ assintomático com todos os pulsos palpáveis e ITB 1,0 bilateral.

Ao concluirmos este artigo, encontrava-se em acompanhamento ambulatorial após 14 meses da intervenção secundária para recanalização do ramo ilíaco da endoprótese. Apresentava-se assintomático, com pulsos universalmente palpáveis e ITB 1,0 bilateral. As angiotomografias de controle realizadas no primeiro, terceiro e sexto mês não demonstraram qualquer sinal de reestenose (Figura 7).

\section{Discussão}

A oclusão de ramo ilíaco de endoprótese aórtica é definida como uma complicação pós CEAAA. Os fatores relacionados à etiologia dessa complicação incluem: áreas de estenose/

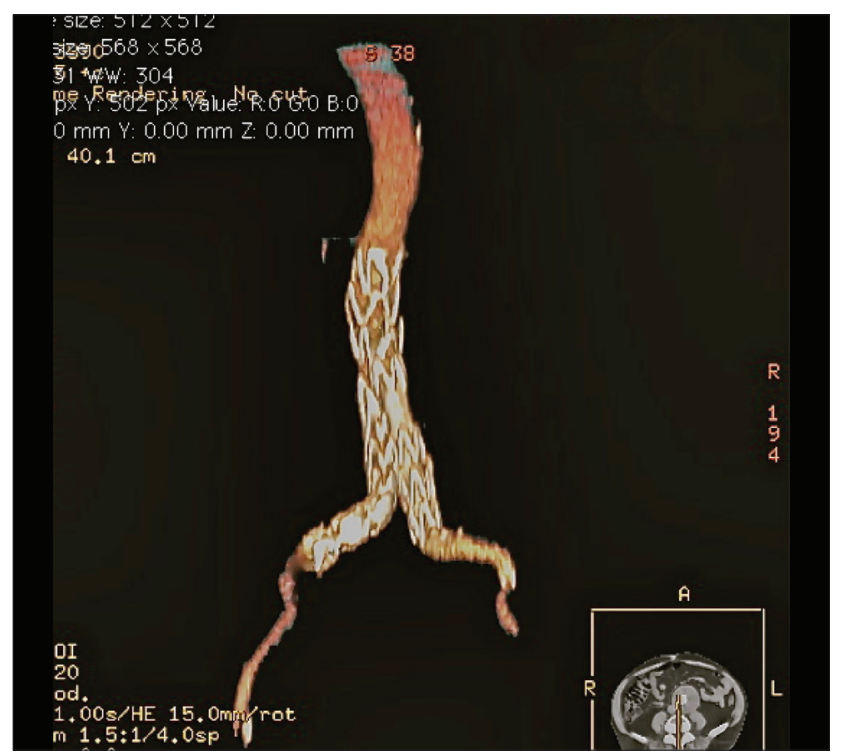

Figura 7. Angiotomografia de controle com perviedade do segmento ilíaco direito recanalizado. 
tortuosidade na artéria nativa ou na endoprótese, leito distal insatisfatório, tipo de endoprótese utilizada (risco elevado em endopróteses de primeira geração e sem suporte), irregularidade na sobreposição dos módulos da endoprótese, extensão da endoprótese até ilíaca externa, diâmetro reduzido das artérias ilíacas, compressão externa e remodelamento arterial ${ }^{16-20}$. Outro fator importante inclui a baixa flexibilidade adotada por alguns tipos de endopróteses, fato este também relacionado aos casos apresentados.

No caso I, em que foi realizado o tratameno de aneurisma sacular infrarrenal e houve evolução com trombose de artéria ilíaca um mês após o tratamento inicial, identificamos a compressão do segmento ilíaco da endoprótese livre de stent (característica já modificada pela Medtronic Inc./USA com a nova endoprótese Endurant ${ }^{\circledR}$ ) pelo ramo ilíaco pérvio contralateral justa bifurcação aórtica (24 mm de diâmetro).

No caso II, com tratamento endovascular de aneurisma infrarrenal de $6,7 \mathrm{~cm}$ de diâmetro e evolução com trombose ilíaca nove meses após o tratamento inicial, identificamos aumento significativo na angulação entre o segmento da fixação distal do ramo ilíaco da endoprótese e a artéria ilíaca comum direita, fato não presente no tratamento inicial.

Acreditamos em remodelamento arterial, visto que não houve desconexão ou migração de nenhum segmento da endoprótese, o diâmetro da artéria ilíaca era superior a $10 \mathrm{~mm}$ e não havia angulação significativa previamente.

Em ambos os casos, a angiotomografia pré-operatória identificou a causa anatômica da trombose, não sendo necessário o uso do ultrassom intravascular (IVUS). Os tratamentos descritos na literatura para essa complicação incluem: trombectomia com cateter de Fogarty, trombectomia com cateter de Fogarty via bainha $16 \mathrm{f}$ seguido de angioplastia com stent primário, trombólise com ou sem implante de stent, trombectomia com sistema reólico Angiojet ${ }^{\circledast}$ (Possis Medical Inc./USA) seguido de implante de stent associado ou não à trombólise, angioplastia com stent primário e procedimentos cirúrgicos abertos anatômicos e extra-anatômicos (ponte femoro-femoral, axilo-femoral e aorto-femoral) $)^{21,22}$.

Vale ressaltar que:

- No caso de trombectomia com cateter de Fogarty, há necessidade de acesso cirúrgico e receio quanto à possibilidade de deslocamento e/ou desconexão da endoprótese, além da causa da trombose não ser tratada ${ }^{23}$;

- A trombólise não é isenta de risco, sendo descrita complicação hemorrágica grave em diversos tipos de tratamento ${ }^{24}$;

- A angioplastia direta sem trombectomia ou trombólise pode levar à embolização de material trombótico, bem como à reestenose precoce;
- Os procedimentos extra-anatômicos apresentam maior morbidade, além de perviedade reduzida a longo $\operatorname{prazo}^{25,26}$.

Optamos pela abordagem inicial totalmente percutânea para o tratamento da oclusão de ramo ilíaco de endoprótese, no caso I, por se tratar de paciente obeso mórbido com riscos elevados de infecção em pontes extra-anatômicas com utilização de próteses na região inguinal, e, no caso II, devido à dificuldade técnica no acesso cirúrgico femoral, pela presença de quelóide na região inguinal direita (paciente submetido à lifadenectomia inguinal por tumor cutâneo), já com acesso cirúrgico prévio à artéria femoral direita. Após identificar a causa anatômica da oclusão, realizamos o acesso percutâneo com introdutores de baixo perfil, seguido de trombectomia rotativa e aspirativa, aspiração esta que acreditamos poder minimizar os riscos de embolia distal, e angioplastia, com stent primário de toda a extensão do segmento ilíaco da endoprótese.

Utilizamos o dispositivo Rotarex ${ }^{\otimes}$ (Straubmedical Inc./ $\mathrm{CHE}$ ), indicado para a remoção de material trombótico agudo ou subagudo em artéria nativa ou no interior de próteses, cujo tamanho varia de acordo com o tamanho do vaso ou da prótese a ser tratada. Ele funciona com um sistema de trombectomia rotacional (40 mil a 60 mil rotações por minuto) associado à aspiração contínua, promovendo o destacamento, a aspiração, a fragmentação e o transporte do resíduo trombótico a um reservatório. $\mathrm{O}$ cateter de trombectomia é manipulado sempre sobre fio guia após a ultrapassagem da oclusão. Suas principais contraindicações incluem: placas calcificadas, posicionamento subintimal do fio guia, impossibilidade de ultrapassar a oclusão pelo fio guia e vasoespasmo contínuo.

O fato de realizarmos a trombectomia somente dentro da endoprótese e essa, nos casos citados, ser composta por exoesqueleto, também nos deu mais segurança para a realização dessa técnica endovascular. No entanto, ressaltamos que o uso do dispositivo apresenta risco de rotura arterial, principalmente em vasos calcificados e de calibre reduzido.

Os procedimentos foram realizados sob anestesia local e sedação, sem necessidade de trombólise ou acesso cirúrgico. $\mathrm{E}$ os procedimentos cirúrgicos extra-anatômicos, na nossa avaliação, devem ficar reservados para os casos de falha no tratamento percutâneo.

O tratamento endovascular com trombectomia percutânea rotativa e aspirativa, seguida de angioplastia com stent primário das oclusões de ramo ilíaco de endopróteses bifurcadas de aorta abdominal, apresenta-se como técnica factível com bons resultados iniciais e baixos índices de complicações operatórias e mortalidade.Todavia, são necessários estudos com maior número de casos, e de um seguimento a mais longo prazo, para 
que a técnica seja recomendada para todos os casos que apresentarem este tipo de complicação.

\section{Referências}

1. Stelter W, Umscheid T, Ziegler P. Three-year experience with modular stent-graft devices for endovascular AAA treatment. J Endovasc Surg. 1997;4(4):362-9. PMid:9418200.

2. Zarins CK, White RA, Schwarten D, Kinney E, Diethrich EB, Hodgson KJ, et al. AneuRx stent graft versus open surgical repair of abdominal aortic aneurysms: multicenter prospective clinical trial. J Vasc Surg. 1999;29(2):292-308. http://dx.doi.org/10.1016/ S0741-5214(99)70382-4

3. Greenhalgh RM, Brown LC, Kwong GP, Powell JT, Thompson SG. Comparison of endovascular aneurysm repair with open repair in patients with abdominal aortic aneurysm (Evar trial 1), 30-day operative mortality results: randomised controlled trial. Lancet. 2004;364(9437):843-8. PMid:15351191.

4. Prinssen M, Verhoeven EL, Buth J, Cuypers PW, van Sambeek MR, Balm R, et al. A randomized trial comparing conventional and endovascular repair of abdominal aortic aneurysms. N Engl J Med. 2004;351(16):1607-18. PMid:15483279.

5. Forbes TL, DeRose G, Kribs S, Harris KA. A cost-effectiveness analysis of standard versus endovascular abdominal aortic aneurysm repair. Can J Surg. 2002;45(6):420-4. PMid:12500916.

6. Mendonça $\mathrm{CT}$, Moreira RCR, Timi JRR, Miyamotto M, Martins $M$, Stanischesk IC, et al. Comparação entre os tratamentos aberto e endovascular dos aneurismas da aorta abdominal em pacientes de alto risco cirúrgico. J Vasc Bras. 2005;4(3):232-42. http://dx.doi. org/10.1590/S1677-54492005000300004

7. Chaikof EL, Blankensteijn JD, Harris PL, White GH, Zarins CK, Bernhard VM, et al. Reporting standards for endovascular aortic aneurysm repair. J Vasc Surg. 2002;35(5):1048-60. http://dx.doi.org/10.1067/ mva.2002.123763

8. Harris PL, Vallabhaneni SR, Desgranges P, Becquemin JP, van Marrewijk C, Laheij RJ. Incidence and risk factors of late rupture, conversion, and death after endovascular repair of infrarenal aortic aneurysms: the EUROSTAR experience. European Collaborators on Stent/graft tecniques for aortic aneurysm repair. J Vasc Surg. 2000;32(4):739-49. PMid:11013038. http://dx.doi.org/10.1067/mva.2000.109990

9. Carpenter JP, Anderson WN, Brewster DC, Kwolek C, Makaroun M, Martin J, et al. Multicenter pivotal trial results of the Lifepath System for endovascular aortic aneurysm repair. J Vasc Surg. 2004;39(1):34-43. http://dx.doi.org/10.1016/j.jvs.2003.10.036

10. Criado FJ, Clark NS, MCKendrick C, Longway J, Domer GS. Update on the Talent LPS AAA stent graft: results with "enhanced talent". Semin Vasc Surg. 2003;16(2):158-65. PMid:12920687.

11. Conner MS 3rd, Sternbergh WC 3rd, Carter G, Tonnessen BH, Yoselevitz M, Money SR. Secondary procedures after endovascular aortic aneurysm repair. J Vasc Surg. 2002;36(5):992-6. PMid:12422089. http://dx.doi.org/10.1067/mva.2002.127966

12. Laheij RJ, Buth J, Harris PL, Moll FL, Stelter WJ, Verhoeven EL. Need for secondary interventions after endovascular repair of abdominal aortic aneurysm. Intermediate-term follow-up results of a European collaborative registry (EUROSTAR). Br J Surg. 2000;87(12):1666-73. http://dx.doi.org/10.1046/j.1365-2168.2000.01661

13. Milner R, Golden MA, Velazquez OC, Fairman RM. A new endovascular approach to treatment of acute iliac limb occlusions of bifurcated aortic stent grafts with an exoskeleton. I Vasc Surg. 2003;37(6):1329-31. PMid:12764286. http://dx.doi.org/10.1016/ S0741-5214(02)75457-8

14. Fairman RM, Baum RA, Carpenter JP, Deaton DH, Makaroun MS, Valazquez OC. Limb interventions in patients undergoing treatment with an unsupported bifurcated aortic endograft system: a review of the Phase II EVT Trial. J Vasc Surg. 2002;36(1):118-26. PMid:12096268. http://dx.doi.org/10.1067/mva.2002.123748

15. Amesur NB, Zajko AB, Orons PD, Makaroun MS. Endovascular treatment of iliac limb stenoses or occlusions in 31 patients treated with the ancure endograft. J Vasc Interv Radiol. 2000;11(4):421-8. PMid:10787199.

16. Chuter TA, Wendt G, Hopkinson BR, Scott RA, Risberg B, Keiffer E, et al. Bifurcated stent-graft for abdominal aortic aneurysm. CardiovasC Surg. 1997;5(4):388-92. PMid:9350793.

17. Carpenter JP, Neschis DG, Fairman RM, Barker CF, Golden MA, Velasquez OC, et al. Failure of endovascular abdominal aortic aneurysm graft limbs. J Vasc Surg. 2001;33(2):296-303. PMid:11174781. http://dx.doi.org/10.1067/mva.2001.112700

18. Boyle JR, Thompson MM, Clode-Baker EG, Green J, Bolia A, Fishwick G, et al. Torsion and kinking of unsupported aortic endografts: treatment by endovascular intervention. J Endovasc Surg. 1998;5(3):216-21. http:// dx.doi.org/10.1583/1074-6218(1998)005<0216:TAKOUA>2.0.CO;2

19. Becquemin JP, Lapie V, Favre JP, Rousseau H. Mid-term results of a second generation bifurcated endovascular graft for abdominal aortic aneurysm repair: the French Vanguard trial. J Vasc Surg. 1999;30(2):209-18. PMid:10436440. http://dx.doi.org/10.1016/ S0741-5214(99)70130-8

20. Chuter TA, Wendt G, Hopkinson BR, Scott RA, Risberg B, Kieffer $E$, et al. European experience with a system for bifurcated stent-graft insertion. J Endovasc Surg. 1997;4(1):13-22. http://dx.doi. org/10.1583/1074-6218(1997)004<0013:EEWASF>2.0.CO;2

21. Silberzweig JE, Marin ML, Hollier LH, Mitty HA, Parsons RE, Cooper $J M$, et al. Aortoiliac aneurysms: endoluminal repair--clinical evidence for a fully supported stent-graft. Radiology. 1998;209(1):111-6. PMid:9769820.

22. Erzurum VZ, Sampram ES, Sarac TP, Lyden SP, Clair DG, Greenberg RK, et al. Initial management and outcome of aortic endograft limb occlusion. J Vasc Surg. 2004;40(3):419-23. PMid:15337867. http:// dx.doi.org/10.1016/j.jvs.2004.06.028

23. Bohannon WT, Hodgson KJ, Parra JR, Mattos MA, Karch LA, Ramsey $\mathrm{DE}$, et al. Endovascular management of iliac limb occlusion of bifurcated aortic endografts. J Vasc Surg. 2002;35(3):584-8. http://dx.doi. org/10.1067/mva.2002.120374

24. Resch T, Lindblad B, Lindh M, Brunkwall J, Ivancev K. Aneurysm expansion and retroperitoneal hematoma after thrombolysis for stent-graft limb occlusion caused by distal endograft migration. J Endovasc Ther. 2000;7(6):446-50. PMid:11194815. 
25. Piotrowski JJ, Pearce $W H$, Jones DN, Whitehill $T$, Bell R, Patt A, et al. Aortobifemoral bypass: the operation of choice for unilateral iliac occlusion?. J Vasc Surg. 1988;8(3):211-8. http://dx.doi. org/10.1016/0741-5214(88)90270-4

26. Ng RL, Gilles TE, Davies AH, Baird RN, Horrocks M. Iliofemoral versus femorofemoral bypass: a 6-year audit. Br J Surg. 1992;79(10):1011-3. http://dx.doi.org/10.1002/bjs.1800791008

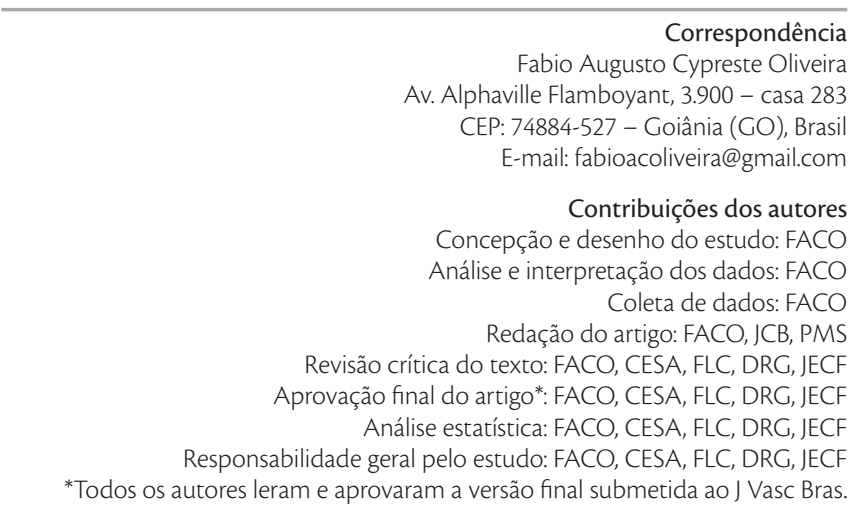

